\title{
LANDSLIDE MONITORING USING TERRESTRIAL LASER SCANNER: GEOREFERENCING AND CANOPY FILTERING ISSUES IN A CASE STUDY
}

\author{
M. Barbarella ${ }^{\text {a, }}$, M. Fiani ${ }^{\text {b }}$ \\ ${ }^{a}$ DICAM, Università di Bologna, Viale Risorgimento 2, 40136 Bologna, Italy - maurizio.barbarella@unibo.it \\ ${ }^{\mathrm{b}}$ DICIV, Università di Salerno, Via Ponte Don Melillo 1 Fisciano (SA), Italy - m.fiani @ unisa.it
}

Commission V, WG V/3

KEY WORDS: LIDAR, TLS, Monitoring, Landslide, DEM/DTM, Reliability, Accuracy

\begin{abstract}
:
In order to define a methodology that faces the major critical issues, we used a Terrestrial Laser Scanner to monitor a large landslide that caused significant disruptions both to an important state road and to a major railway line in Italy. To survey the landslide we used three different models of Terrestrial Laser Scanners, including a "full wave form" one, potentially useful for filtering vegetation from the data. The output of each measurement campaign is a Digital Surface Model referred to a unique reference system. Starting from the DSMs we produced the Digital Terrain Models, one for each survey. The use of different models of TLS together with the software packages recommended by the companies for data processing, allowed us to compare the surveys and to evaluate the reliability and the accuracy of results. The comparison of data has been useful in order to identify and analyse over time the areas of greatest deformation and the directions of landslide movement and it also gives us some elements about the validity of the technique in this kind of applications. The laser surveys have shown a strong dynamic of the slope but have also highlighted some difficulties in order to efficiently filtering the data. Using two different kinds of TLS, full wave form and mono eco, on the same portion of landslide allows us to make comparisons between the two methodologies for landslide monitoring in a real-world context.
\end{abstract}

\section{INTRODUCTION}

In the analysis of landslides (predicting, monitoring and alerting for early warning, monitoring for change detection, etc.) a variety of geomatic techniques may be used, going from the most effective established techniques (use of RT GNSS receivers, Total Station and levelling surveys when applicable ) to the most innovative ones like SAR interferometry (Pieraccini et Al., 2006) Airborne Laser Scanning, remote sensing.

All of them present some critical aspects and strengths as far as landslide monitoring applications are concerned. Some difficulties in surveying a landslide can be caused by the area orientation, the slope gradient, the presence of canopy and the visibility from the stable points.

In order to detect large surfaces one should use a variety of techniques, from the most efficient ones for a global representation of the whole area to those that enable highprecision measurements of single points or areas. For repeated measurements of small areas some methodologies are nevertheless very ineffective. Among the suitable techniques, the Terrestrial Laser Scanner (TLS) is one of the most promising and most generally applicable (Prokop and Panholzer, 2009). The Terrestrial Laser Scanners allow us to survey in a very fast, efficient and precise way a number of buildings and industrial structures, which have a well-defined geometry and which can eventually be modelled to a known shape. They are very useful because of their ability to describe in details the geometry of objects, and are also used for surveying statues and archaeological sites.

In the case of terrain surveys (for example landslides and quarries) characterized by a very complex shape and which are not mathematically modelled, however, one can encounter problems both in the surveying and in the raw data processing. The visibility between the instrument and the ground is often limited due to terrain morphology (vertical and horizontal undulations, steps), making it difficult to identify the laser station points. In addition, the soil is often covered with vegetation and trees: the latter create shadow zones of the ground making them more difficult to detect. The presence of low vegetation and grass makes it very difficult to distinguish the true ground surface within the laser point clouds.

It can be therefore troublesome to extract the DTM from the DSM: this reduces the reliability and the accuracy of the survey. This problem can have negative effects on the reliability of the survey especially in the case of the monitoring of ground deformation, performed through the comparison of repeated surveys carried out at regular intervals. To estimate the shape change of a landslide or of a quarry and which portion has remained stable, and to determine the amount of material that has moved or has been removed is here a problem.

The comparison is performed on the surfaces reconstructed by interpolating the point clouds; we suppose that such surfaces represent the shape of the ground at the time of that particular survey. If the vegetation filtering has not been successful and the DTM has not been properly rebuilt, the comparison is not reliable because it takes into account the change in vegetation occurred in the period between the two surveys.

Another problem which is not always adequately considered and solved is the need for a reference system that remains very stable over time, where the point positions measured in repeated surveys may be framed. In order to compare two surveys carried out at different times it is mandatory that both surveys have been framed in the same reference system; if this does not remain stable over time, the movement of the reference points leads to a wrong estimate of the positions of the laser station and of the point clouds. The reconstructed surface will be traslated with respect to what it should be.

The comparison of the surfaces coming from the two surveys will show fictitious strong variations. Nevertheless the LiDAR technique could be a viable alternative to the traditional surveying methods in surveying large areas and producing a $3 \mathrm{D}$ model of the landslide slope in a direct way. 
When we apply the technique to a real case a lot of difficulties arise, due to the nature of sites, to the large size of the area to survey, to the great distance of the station points from the sliding slope, to the presence of canopy and finally to the need to establish a reference frame for surveys that is stable in time.

\section{SURVEYING METHODOLOGIES}

The numerical modelling of a landslide from a point cloud obtained with a terrestrial laser scanner can be difficult in presence of vegetation: for example trees can hide the ground for an extent that depends on the beam inclination with respect to the landslide surface: this makes difficult the reconstruction. Grass and shrubs also make difficult to distinguish the ground surface from the vegetation. The morphology of the terrain (slope, ripples) limits the areas where you can set up the TLS. Often the laser beam is slightly tilted with respect to the ground surface with dangerous effects on the perspective one obtains.

When planning the survey the choice of best tool depends essentially on the maximum distance between the instrument and the ground: the minimum step of the angular increases and the acquisition frequency are less important, since all the modern instruments offer excellent performances.

The number of points acquired for surveying in a fast way vast areas is very high, and can cause problems during processing: often the first process step is thus thinning the point clouds. The choice of a wide range instrument is not a problem since some vendors offer TLS normally with a range of several hundred meters and even of one or two kilometres. With regard to the choice of the level of performance of the instrument, a significant improvement can instead be given by using a "full wave" instrument (Mallet and Bretar, 2009), that discretizes the TLS return waveform in order to identify the various obstacles hiding the course of the laser beam in a given direction at different distances: this helps filtering the noise introduced by the vegetation using the appropriate software tools. We have used three different TLS instruments, one of which "full wave", to verify whether in a medium distance terrestrial survey it could give some advantages over other instruments in filtering data. When the purpose of a TLS survey is to monitor ground deformations it is essential to identify a set of points that are stable in time and can form the fixed reference system in which a number of repeated measurements made at different moments time can be framed. Such fixed points (we will refer to them as "near reference ") should be placed outside the monitoring area, far enough not feel the effects of the phenomenon one is studying, but close enough to make easy and accurate the connection between them and both the laser station and the target points. The laser station must be placed as close as possible to the surveying area, in a frontal position, and it must be completely visible full. Because of this it cannot be always set up on a stable area: this is even more true for the targets that are used for recording and georeferencing the data. Therefore, in each survey we have measured both the positions of station points and the target ones. The well-materialized points, stable in time, that implement the "near reference", can be placed at a larger distance: they do not need to be visible from the surveying area. The connection with all or some of the targets and station points it is generally possible by means of GPS receivers. If some targets are in a position that does not allow the GPS survey (for example close to a steep slope), their position can be measured using a Total Station, starting from the station points (from which they are certainly visible), oriented in the direction of other points measured with the GPS. The connection between the "near reference" points and the landslide ones can also be done using Real Time Kinematic
(RTK) methodology, which assures a higher execution speed and a precision that, even if is lower than the one achievable with static GPS baselines, can be surely an acceptable survey methodology. Given the importance of ensuring the stability of the reference system used in the surveys done at different times it is also appropriate that the points of the "near reference" are themselves monitored. In order to keep the cost down and to achieve high accuracies one should connect these points with permanent GNSS stations, which are now widespread on Italian territory, on these "near reference" points (this is done in addition to the use of GPS receivers).

The baselines between the reference points and the Permanent Stations (PS) are determined by measurements of long duration taken on the reference points: this is also needed when surveying a landslide site. The data needed to characterize the PS data are generally free for post processing. The time one needs to station on the reference points depends on the distance between them and the PS: typically it is appropriate to leave the receiver on a reference point for the full working day, even when the measurements related to the landslide site are shorter. This solution also involves the choice of a geodetic system.

The PS are generally defined in a Terrestrial Reference System such as the ITRS (current frame ITRF08). In this reference system their coordinates vary over time due to the motion of the plate to which they belong; in Europe the Euro-Asian plate moves about $2 \mathrm{~cm} / \mathrm{y}$ with respect to the ITRS. To make the PS coordinates more stable over time, a Geodetic System defined exclusively by reference stations materialized on stable part of the plate (in Europe this is the ETRS89) should be used.

We can conclude that the geodetic system ETRF89 (the current frame is the ETRF2000), that guarantees over time very small movements (a few $\mathrm{mm} / \mathrm{y}$ ) of the reference stations used for the laser scanner survey, should be used for monitoring purposes. With regard to the coordinate system to be adopted for laser data processing, it should be noted that more TLS stations are often needed in case of vast areas, and it may be necessary to take into account the curvature of the earth.

In TLS survey each station refers to a set of three dimensional orthogonal Cartesian system where the coordinates of the points belonging to the clouds are calculated starting from the measured $3 \mathrm{~d}$ polar coordinates (azimuthal and zenital angles, radial distance). This triad has an axis ( $\mathrm{Z}$ ) along the vertical direction and the local XY plane on the horizontal plane. For point clouds georeferencing we must then perform a transformations between the geocentric system where GPS measurements are defined and the instrumental system. Since a geocentric system is not adequate to describe in a immediately intuitive way the altimetric trend of the ground, it may be suitable to use a cartographical representation for the planimetric component and the ellipsoidal height for altimetry position of the points.

In case of ground deformation monitoring is not necessary to apply the geoid undulation in order to express the data as orthometric heights, since the correction is constant in time. In our tests we have implemented a number of software tools for the transformation of the points of the clouds from a type of coordinates to another one (geocentric, ellipsoidal, local geodetic, cartographic). In order to define a methodology that could overcome the major critical issues, we used a TLS to monitor a large landslide (more than 10 ha) that caused significant disruptions both to an important state road and to a major railway line in Italy. As far as the surveying methodology is concerned, in the attempt to filter as effectively as possible the vegetation, we used three different models of Terrestrial Laser Scanner: we selected two long range models (Optech ILRIS $36 \mathrm{D}$ and Riegl VZ-400) and a $150 \mathrm{~m}$ range one (Leica C10) that we used in the lower part of the landslide, where the 
distance between the station point and the landslide slope is smaller. One of them is a "full wave" instrument and has been used to test whether this technology is able to give significant improvements. In the inferior part of the landslide, during the same campaign, we used in rapid succession the Riegl VZ400 and the Leica $\mathrm{C} 10$ in order to make a direct comparison between the two instruments.

As for the survey frame:

- the target and the TLS stations have been surveyed by GPS baselines referred to the "near reference";

- the "near reference" consists in two pillars materialized on a concrete wall in the stable area located at walking distance from the survey one;

- the two pillars were themselves connected to two PS belonging to a NRTK Network (Network Real Time Kinematic) for real time surveying service of the Campania Region, far 26 (Castellabate) and 38 (Sapri) $\mathrm{km}$ away from the landslide. In view of the distance between the landslide area and the PS, we made continuous GPS observations during two consecutive days. We show the scheme of the "stable frame" in figure 1.

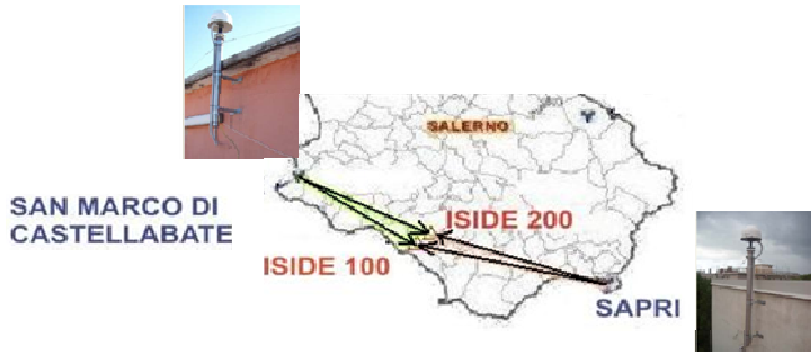

Figure 1. RTK Permanent GNSS Stations (reference frame)

Once the coordinates of the permanent stations were fixed in the ETRF2000 system, we calculated the adjusted positions of the two pillars (we used the mean value of two survey days). Starting from these two pillars, which are considered as fixed, we finally calculated the coordinates both of the target and the station points, always in the European System

\section{LANDSLIDE DESCRIPTION AND FIRST SURVEYS}

The phenomenon we are monitoring is taking place in Pisciotta (Campania Region, Italy), on the left side of the final portion of a stream ("Fiumicello"), and has caused significant disruptions both to an important state road and to a major national railway line. The landslide has a very large size (more than $10 \mathrm{ha}$ ) and affects a stretch of road and two railway tunnels with heavy traffic (in both North-South and South-North directions). In 2008 mud and soil brought by the landslide into the river after heavy and prolonged rains has caused the blockage of both tunnels and the interruption of rail traffic on the main northsouth railway line in Italy for about one day. The landslide is active and is defined "type slow" according to the classification of Cruden and Varnes (Cruden and Varnes, 1996). The movement of the soil is of the sliding rotational type, with the typical slipping that occurs along deep surfaces. In figure 2 we show the landslide and an extract of the corresponding geological map (Guida and Siervo, 2010); the rocks are a mix of "marnoso-arenacee", calcareous and clayey of the Cilento flysch. The first survey of the phenomenon has been carried out by the Iside srl group (Iside, 2007): it has set up a system mainly oriented to the monitoring of the area close to the road. The system was basically done from a number of sensors, like fixed weather stations, piezometers, inclinometers, strain gauges and from a topographical network built upon:
- two fixed points placed on the "stable" side of the mountain and materialized with pillars where a total station was placed; - about fifty vertices materialized permanently with metal rods; when measuring retro-reflective micro prisms are mounted on the rods: the micro prisms can be measured from the two fixed points with the Total Station;

- six vertices that can be measured using GPS (since they are not visible from the two fixed points).
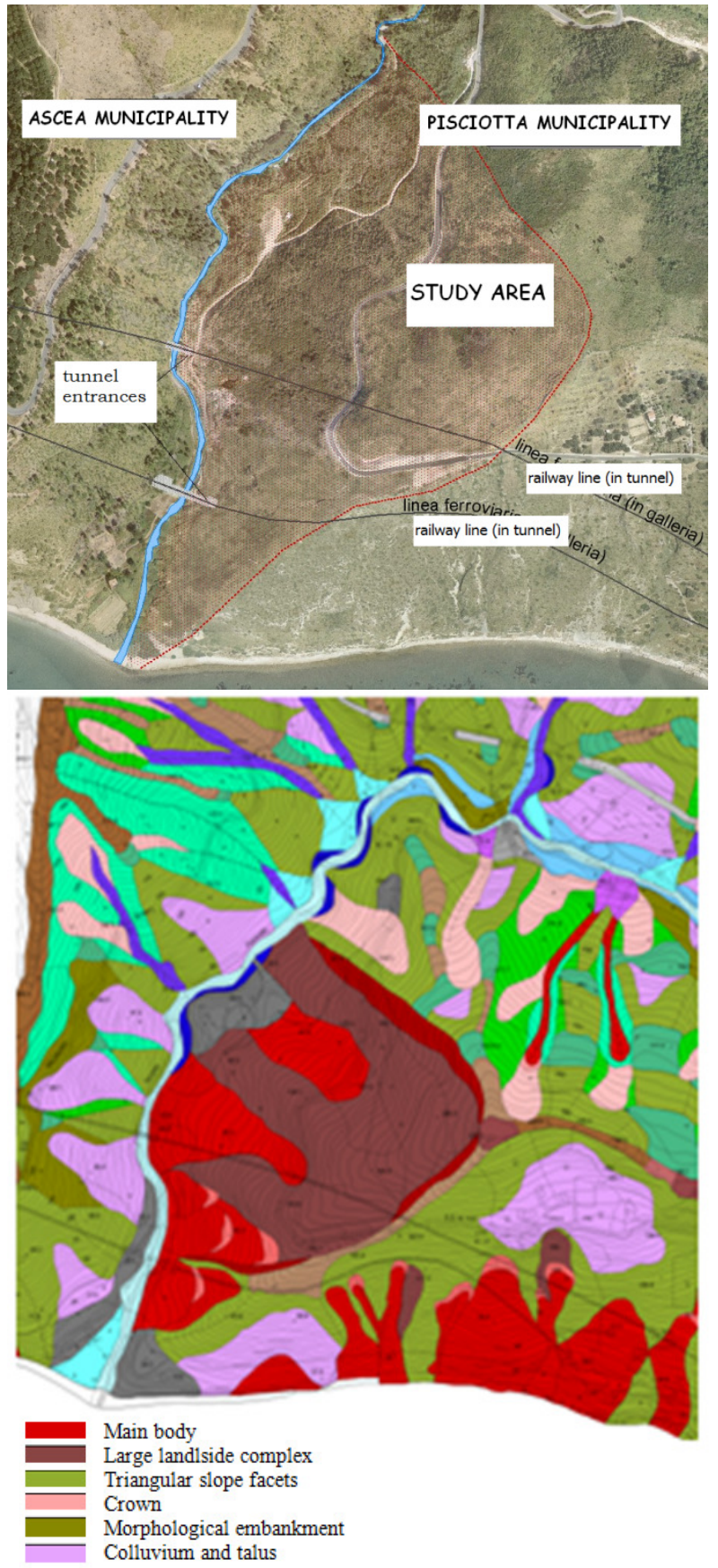

Figure 2. Location of landslide and extract of geological map

Since older topographical measurements or older data are not available, and since we only have measurements on individual points, it is impossible to compute reliably the volume of the moving material or to define precisely the area affected by landslide. It is however possible to determine reliably and accurately the movements of the individual observed points and 
in this way to obtain a precise information on the dynamics of the phenomenon. For all points of the topographical network there are graphs that show the trend of the planimetric and altimetric displacements and the related velocity in the period 2005-2009. The results of the periodic measurements show that the planimetric displacements in a period of about 4 years has been of around $7-8 \mathrm{~m}$ while the altimetric movement has been approximately of about $2.5-3.5 \mathrm{~m}$. The average daily speed of the landslide was found to be of approximately $0.5 \mathrm{~cm} /$ day with peaks of up to $2 \mathrm{~cm} /$ day in planimetry and of about $0.2 \mathrm{~cm} /$ day with peaks up to $1.5 \mathrm{~cm} /$ day in height. The consequences of these movements are visible on a long stretch of road that appears to be completely disrupted (figure 3 ).

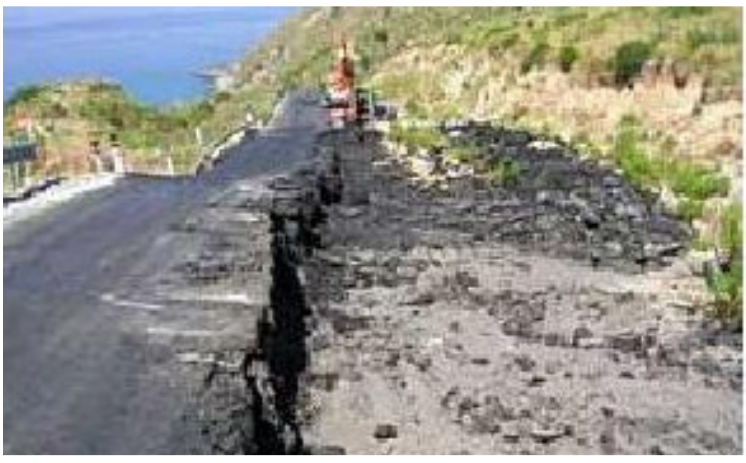

Figure 3. Deep cracks in the road

\section{SURVEYING CAMPAIGNS}

The rugged morphology of survey locations naturally was a strong conditioning element in the choice of the measurement type. The strong landslide dynamics requires the surveying campaigns to be carried out under tight deadlines. The TLS and GPS surveys had therefore to be carried out in the same day.

We carried out a LiDAR "zero" measurement and about three months later a repetition measurement using a long-range terrestrial laser scanner, Optech Ilris 36 D. In order to survey the top of the landslide area the laser stations can be located in a few points on the stable side facing the landslide at several hundred meters away from the area. A further measurement campaign was carried out in the summer of 2011. In the latest survey we also used a laser instrument capable to analyze the calibrated relative reflectance in the attempt to filter the effect of the obstacles (for instance the canopy) and thus to generate easier a DTM starting from the DSM acquired. In the third campaign we used a Riegl VZ-400 instrument for surveying the upper part of the landslide, the most troubled due to the presence of canopy, and a Leica C10 in the lower part where natural ground motion and man-made actions have bared the soil making easier the shape reconstruction. The location of the seven TLS stations, the spherical targets we used, and the Iside vertices connected to the PS network are shown in figure 4.

In all survey campaigns we collected laser scan measurements from at least two station points, located on the stable slope opposite to the landslide: the two station points were at different altitudes. From these stations the laser scan measurements were collected both in the upper part and in the lower part of the landslide, down to the stream. In each scan we use a number of targets (figure 5). As we said, in order to frame the whole survey, the coordinates were measured by GNSS instruments in static mode keeping fixed the vertices Iside100 and Iside200. We used different types of targets, both spherical and of the type suggested by the software vendors (cylindrical and plane) for easy recognition when using the processing software Cyclone and RiscanPro. The "final" coordinates of the targets came from a least squares adjustment in ETRF2000; we eventually transformed them into other coordinate systems (local geodetic having Iside100 as origin, UTM and Gauss).

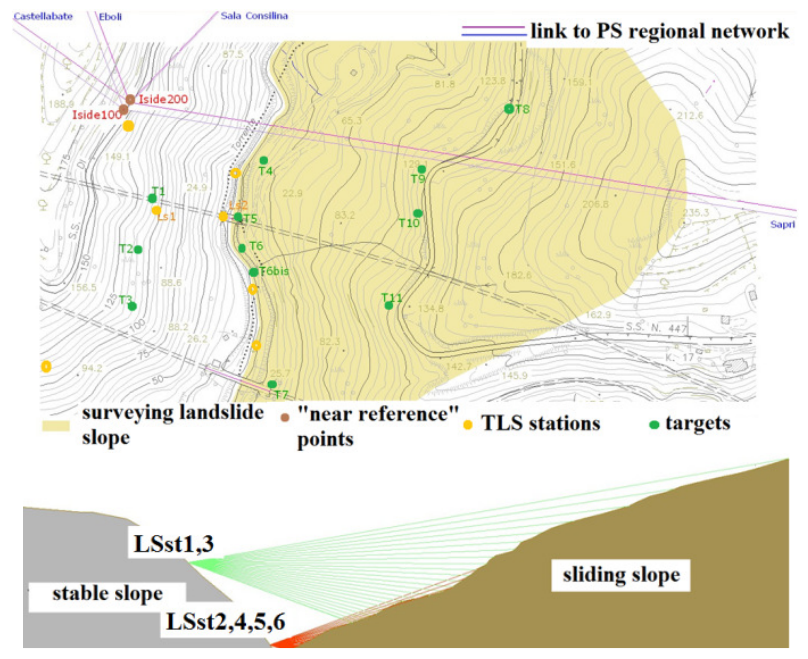

Figure 4. Planimetric e altimetric schemes of the last survey

\begin{tabular}{|c|c|c|c|c|}
\hline Campaign & $\begin{array}{c}\text { TLS } \\
\text { instrument }\end{array}$ & Tilt & $\begin{array}{c}\text { scans } \\
\text { number }\end{array}$ & $\begin{array}{c}\text { points } \\
\text { number }\end{array}$ \\
\hline Feb'10 & $\begin{array}{c}\text { Optech } \\
\text { Ilris 36 } D\end{array}$ & $-20^{\circ},-15^{\circ}, 0^{\circ},+15^{\circ}$ & 18 & $15^{*} 10^{6}$ \\
\hline Jun'10 & $\begin{array}{c}\text { Optech } \\
\text { Ilris 36D }\end{array}$ & $-30^{\circ},-20^{\circ}, 0^{\circ}$ & 22 & $17^{*} 10^{6}$ \\
\hline Jun'11 & $\begin{array}{c}\text { Leica C10 } \\
\text { Riegl VZ400 }\end{array}$ & - & 5 & $108^{*} 10^{6}$ \\
\hline
\end{tabular}

Figure 5. Synthesis of surveys

\section{RESULTS AND CONCLUSIONS}

All the scans have been edited, one by one, and they have been aligned among them in order to produce a single global scan for each of the three surveys we carried out. Finally we have georeferenced the outputs by using the targets coordinates. For editing, registration and georeferencing we have used the Polyworks tool and the two ones recommended by the manufacturers of the TLS we used: RiscanPro (Riegl) and Cyclone (Leica). The process of registration and georeferencing has always lead to low values of residues: this suggests we have reached a good precision (eg georeferencing done with Polyworks is characterized by a mean absolute value of $5.7 \mathrm{~cm}$ ). Figure 6 shows a part of the point cloud obtained by using the Leica ScanStation C10: here we are in the lower part of the landslide, close to the stream. There have been some problems with the data obtained with the Optech TLS: the instrument base rotates to make obtaining a complete scan possible. In figure 7 we show with different colors the different parts of the point cloud that have been observed in each position of the rotating base (the scan that we are showing is based on eight different positions of the basis). The different frames that all together form the complete scan are not always correctly oriented. To obtain data of good quality it has been necessary to calibrate the rotating base (this had to be done from the Optech engineers). In the table of figure 7 we give in meters some parameters relating to registration (reg) and georeferencing (georef). 


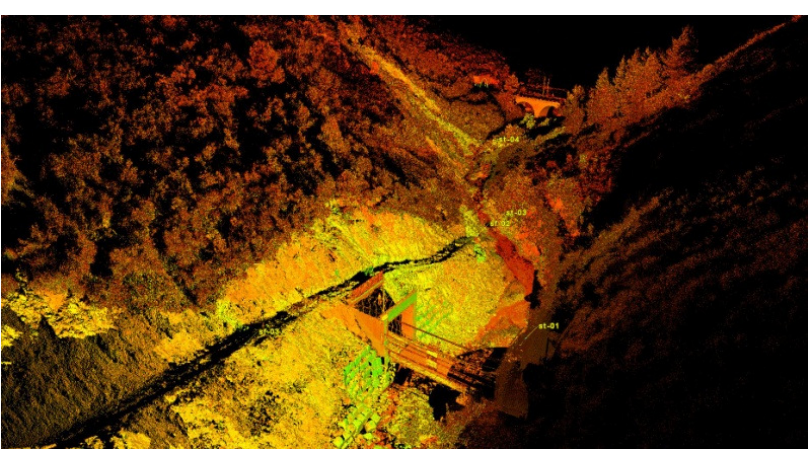

Figure 6. Point clouds, third survey (2011)

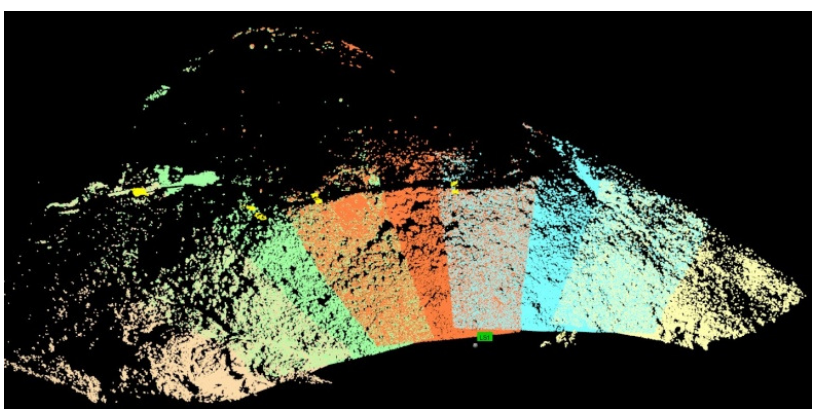

\begin{tabular}{|c|c|c|c|c|}
\hline $\begin{array}{c}\text { Survey } \\
\text { campaing }\end{array}$ & $\begin{array}{c}\text { Max error } \\
(\mathrm{m})\end{array}$ & $\begin{array}{c}\text { Min error } \\
(\mathrm{m})\end{array}$ & $\begin{array}{c}\text { St Dev } \\
(\mathrm{m})\end{array}$ & Operation \\
\hline 1 & 0.3 & -0.3 & 0.08 & reg \\
\hline & 0.015 & 0,007 & 0.004 & georef \\
\hline 2 & 0.2 & -0.2 & 0.03 & reg \\
\hline & 0.062 & 0.033 & 0.011 & georef \\
\hline
\end{tabular}

Figure 7. A single point cloud acquired with Optech

Once these steps have been done a number of DTMs of the landslide are available. Since all surveys have been framed in the same reference system, it is possible to compare the DTMs obtained at different times, by interpolating the point clouds on a grid: this allows to identify and analyse how the areas with largest deformation evolve in time, and to estimate the directions of landslide movement.

In order to obtain a detailed comparison between different epochs, we have worked on small portions of the area: this has made the computational problem far easier to deal with. The different portions have been selected by assuming geological homogeneity of the terrain but also the presence of useful details (probably) stable over time: we expect that both the upstream and the downstream railways tunnels, clearly visible on the scans, should not have moved significantly in the time elapsed between the two surveys.

The comparison of data coming from the first two campaigns gives us some elements about the validity of the technique in this kind of applications. The laser surveys have shown a strong dynamic of the slope. Figure 8 shows an example of the analysis: the magnitude of the deplacment is represented in a chromatic scale. We have found that the displacement among two successive surveys can reach the value of $50 \mathrm{~cm}$. This estimate is in agreement with what measured at individual points by Total Station from the ISIDE group (Iside, 2007) in previous years (2004-2009), and indicates a stable trend, that continues regularly. The laser scanner survey allows to map the behavior of different areas, provides a much more complete and distributed information and facilitates the interpretation of the local geo morphology.

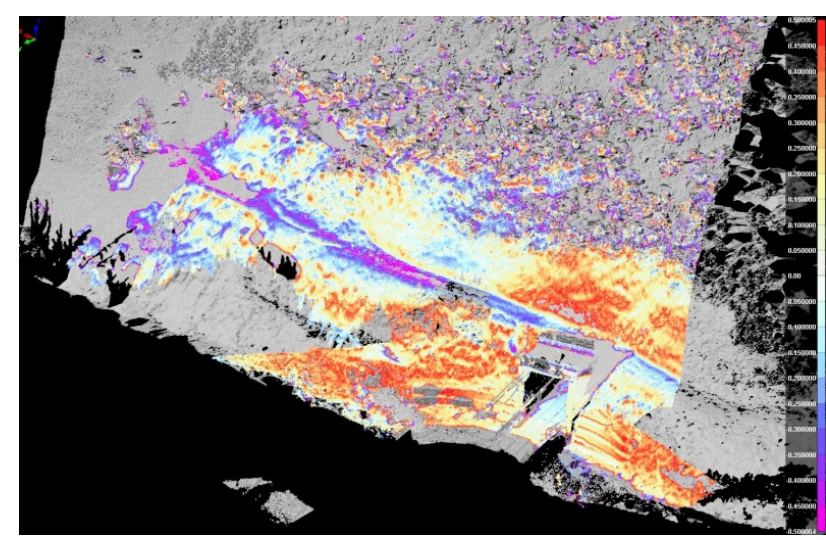

Figure 8. Comparison between two successive surveys

We have also been able to compare the performances of different instruments. In figures 9,10 and 11 we show the point clouds detected by $\mathrm{C} 10$ and VZ400 on the same area, and the difference obtained by considering the residuals of the points of a first scan with respect to the surface obtained by interpolation of the cloud measured in a second scan. Data editing is not yet perfect, but already at the scale of the figure we can see that in areas with poor or no vegetation (the lower part of the landslide on east side and along the road) the height differences are very regular, while in the higher part, where there is more vegetation, the differences vary irregularly and have to be considered not significant before of a detailed editing of the vegetation.

The presence of vegetation is not the only one problem of the data. Figure 12 shows an effect not yet fully explained, which leads to errors that are very clear close to the tunnel: the laser stations were placed exactly at the tunnel entrance, and the response of the internal part of the tunnel, not optically visible, produces signals which are interpreted by the software of both instruments as points located at a completely wrong height. This effect is not present in the first two surveys, when the TLS stations were placed slightly aside the tunnel entrance, not in axis with the tracks.

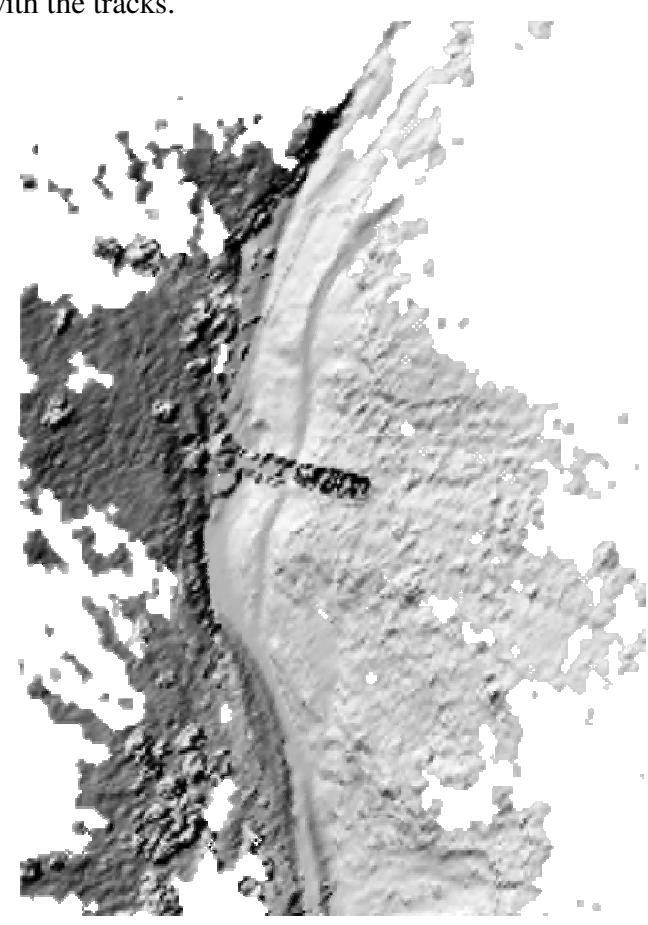

Figure 9. Leica survey 


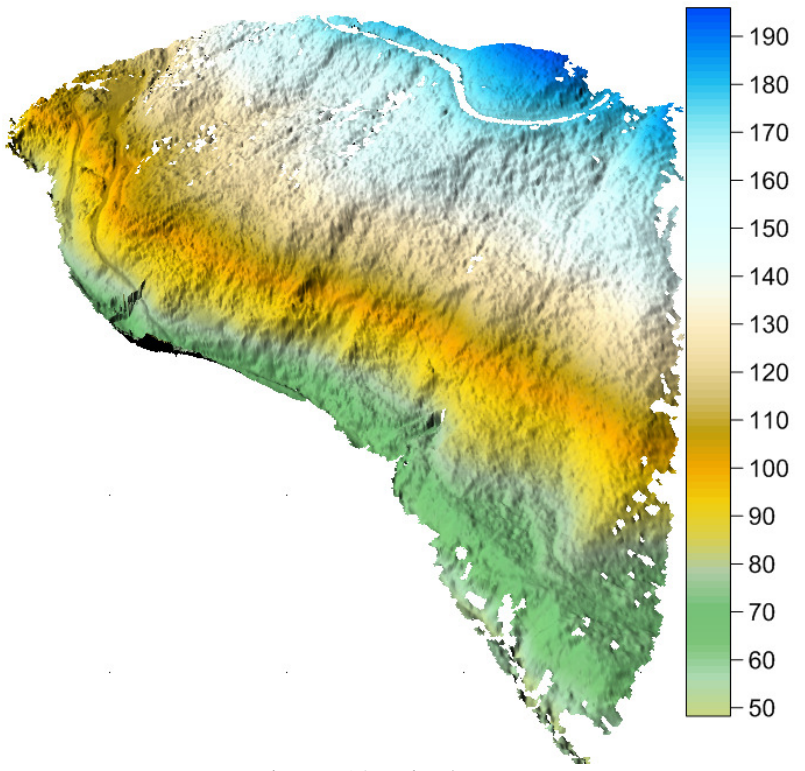

Figure 10. Riegl survey

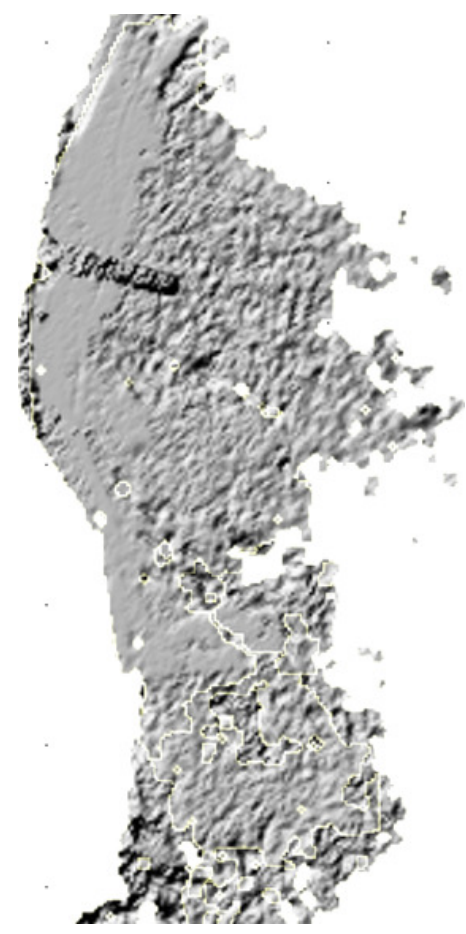

Figure 11. Comparison between Riegl and Leica point clouds

The TLS allows to acquire in a short time the position of a huge amount of points of the ground and therefore makes possible to describe the entire surface of interest and not only some isolated points. It is very important to link the survey to a frame that is stable in time: this allows to monitor the variation of the shape of the landslide.

The link to Permanent GNSS Stations and the use of a Geodetic Reference Frame like ETRF2000 allows to solve the problem without a significant increase of field work.

The editing of the data to reduce noise, first among all the one induced by the vegetation, requires a large amount of work: without this it is not possible to use the data for a quantitative precise analysis of the situation. There are also some effects that are difficult to explain as only determined from the shape of the ground, although they can be filtered out easily due to their size.

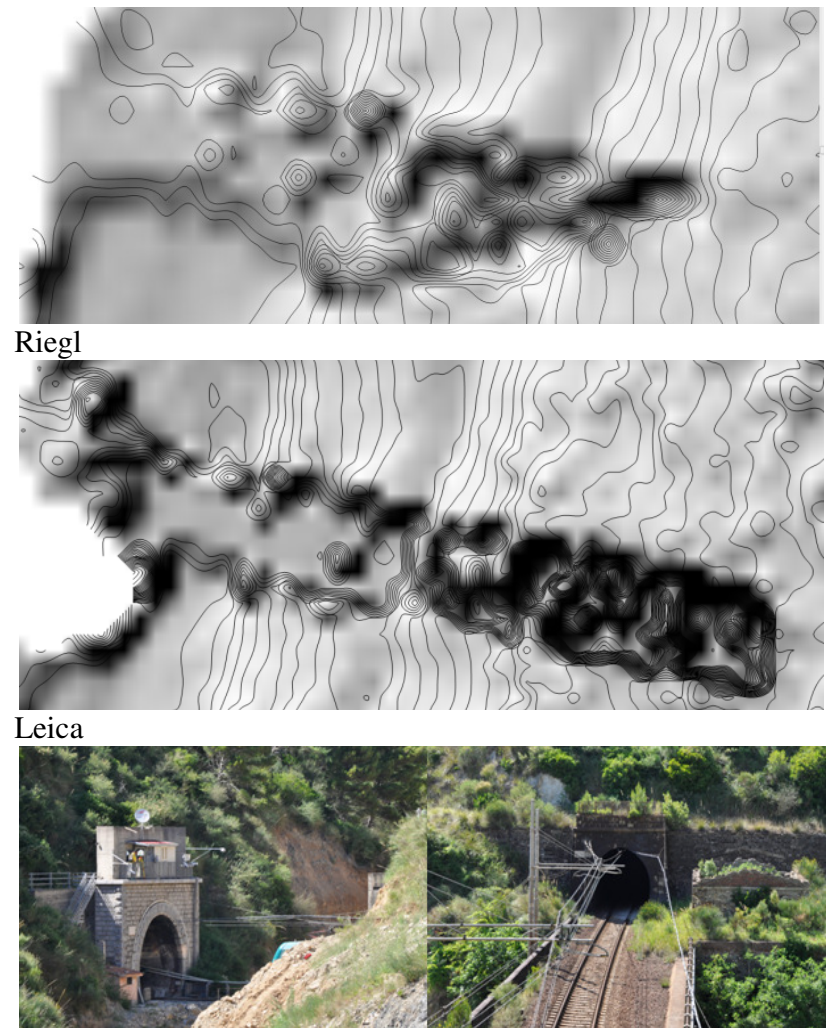

Figure 12. An unexplained effect of the railways

\section{References}

Cruden, D.M., Varnes, D.J., 1996. Landslides Types and Processes. Landslides: Investigation and Mitigation, Turner A.K. \& Schuster R.L. Eds., Transportation Research Board Special Report 247. National Academy Press, WA, pp.36-75.

Guida D., Siervo, V., 2010. In: Seminar on Landslides in Structurally Complex Formations, Geologist Regional Ass. in Salerno.

Iside S.r.l., 2007. Servizio di monitoraggio dei corpi franosi insistenti sulla ex s.s. n. 447 tra km 70 e 72 nel comune di Pisciotta. http://www.centroiside.net (accessed 20 Apr. 2012).

Mallet C., Bretar, F., 2009. Full-waveform topographic Lidar: State-of-the-art. ISPRS Journal of Photogrammetry and Remote Sensing, 64, pp 1-16.

Pieraccini, M. , Noferini, L. , Mecatti, D. , Atzeni, C. , Teza, G., Galgaro, A., Zaltron, N., 2006. Integration of Radar Interferometry and Laser Scanning for Remote Monitoring of an Urban Site Built on a Sliding Slope. IEEE Transactions on Geoscience and Remote Sensing, 44(9).

Prokop, A., Panholzer, H., 2009. Assessing the capability of terrestrial laser scanning for monitoring slow moving landslides. Nat Hazard Earth Sys, 9, pp. 1921-1928.

\section{Acknowledgements}

We thanks Giovanni Abate from Leica Instruments for Leica C10 ScanStation lending and for the support they gave us in 2011 surveying campaign. 